\title{
Magnetic resonance imaging of intraocular optic nerve disorders: review article
}

\author{
Tomonori Kanda ${ }^{1, A, B, E, F}$, Aki Miyazaki ${ }^{1, E}$, Feibi Zeng ${ }^{1, E}$, Yoshiko Ueno ${ }^{1, E}$, Keitaro Sofue ${ }^{1, E}$, Takaki Maeda ${ }^{1, E}$, \\ Munenobu Nogami ${ }^{1, \mathrm{E}}$, Kazuhiro Kitajima ${ }^{2, \mathrm{E}}$, Takamichi Murakami ${ }^{\mathrm{1}, \mathrm{E}}$ \\ 'Department of Radiology, Kobe University School of Medicine, Kobe, Japan \\ 2Department of Radiology, Division of Nuclear Medicine and PET Centre, Hyogo College of Medicine, Nishinomiya, Japan
}

\begin{abstract}
The optic nerve is morphologically classified as a peripheral nerve, but histologically it shares characteristics with the central nerves. Diseases that affect vision and the optic nerve are many and varied: optic neuritis, demyelination (multiple sclerosis, acute disseminated encephalomyelitis, neuromyelitis optica spectrum disorder, and myelin oligodendrocyte glycoprotein antibody-related disorders), drugs, collagen disease, vasculitis, infection, trauma, vascular abnormalities, tumours, and non-tumoural masses. In this review, we summarise the magnetic resonance imaging findings for various pathological conditions that cause deterioration in visual acuity.
\end{abstract}

Key words: magnetic resonance imaging, optic nerve, optic nerve disorders, intraorbital disorders, paraorbital disorders.

\section{Introduction}

The optic nerve is a relatively small structure, with a total length of $50 \mathrm{~mm}$, but there are numerous causes of optic nerve disorders, including: inflammation, tumours, vascular disorders, metabolic diseases, infections, drugs, systemic diseases, genetic diseases, and trauma (Table 1). The frequency of each disorder is relatively low, and it is difficult to differentiate between them in daily practice. Magnetic resonance imaging (MRI) is a common diagnostic imaging method for optic nerve disorders. However, abnormal findings for the optic nerve on MRI are similar and nonspecific for these diseases. Optic nerve enlargement with high signal intensity on $\mathrm{T} 2$-weighted images and contrast effect in the optic nerve are the main findings with optic nerve abnormalities. It is necessary to distinguish between abnormal findings around the optic nerve and clinical findings to differentiate these diseases $[1,2]$. In this paper, we review the imaging findings and clinical background of various diseases that cause optic nerve abnormalities.

\section{Anatomy}

The optic nerve is commonly divided into four parts: intraocular, intraorbital, optic canal, and intracranial. In the orbit, the optic nerve is surrounded by meninges called the optic nerve sheath, which extends from the intracranial meninges to the eye. Cerebrospinal fluid exists between the optic nerve sheath and the optic nerve and is continuous with the intracranial arachnoid space [2]. The blood supply of the optic nerve comes mainly from the central retinal artery, which is a branch of the ophthalmic artery. The central retinal artery enters the optic nerve approximately $1 \mathrm{~cm}$ behind the eye $[1,2]$.

\section{Magnetic resonance imaging protocols}

Magnetic resonance imaging protocols for optic nerve evaluation vary from report to report, and there is no single definitive protocol. Axial and coronal T1-weighted images without fat suppression, axial and coronal short-T1 inversion recovery (STIR) images, and axial and coronal

\section{Correspondence address:}

Tomonori Kanda, Department of Radiology, Kobe University School of Medicine, 7-5-2, Kusunoki-cho, Chuo-ku, Kobe, Hyogo, 650-0017, Japan, e-mail:k_a@hotmail.co.jp

Authors' contribution:

A Study design · B Data collection · C Statistical analysis · D Data interpretation · E Manuscript preparation · F Literature search · G Funds collection 
Table 1. Differential diagnosis of optic nerve disorders

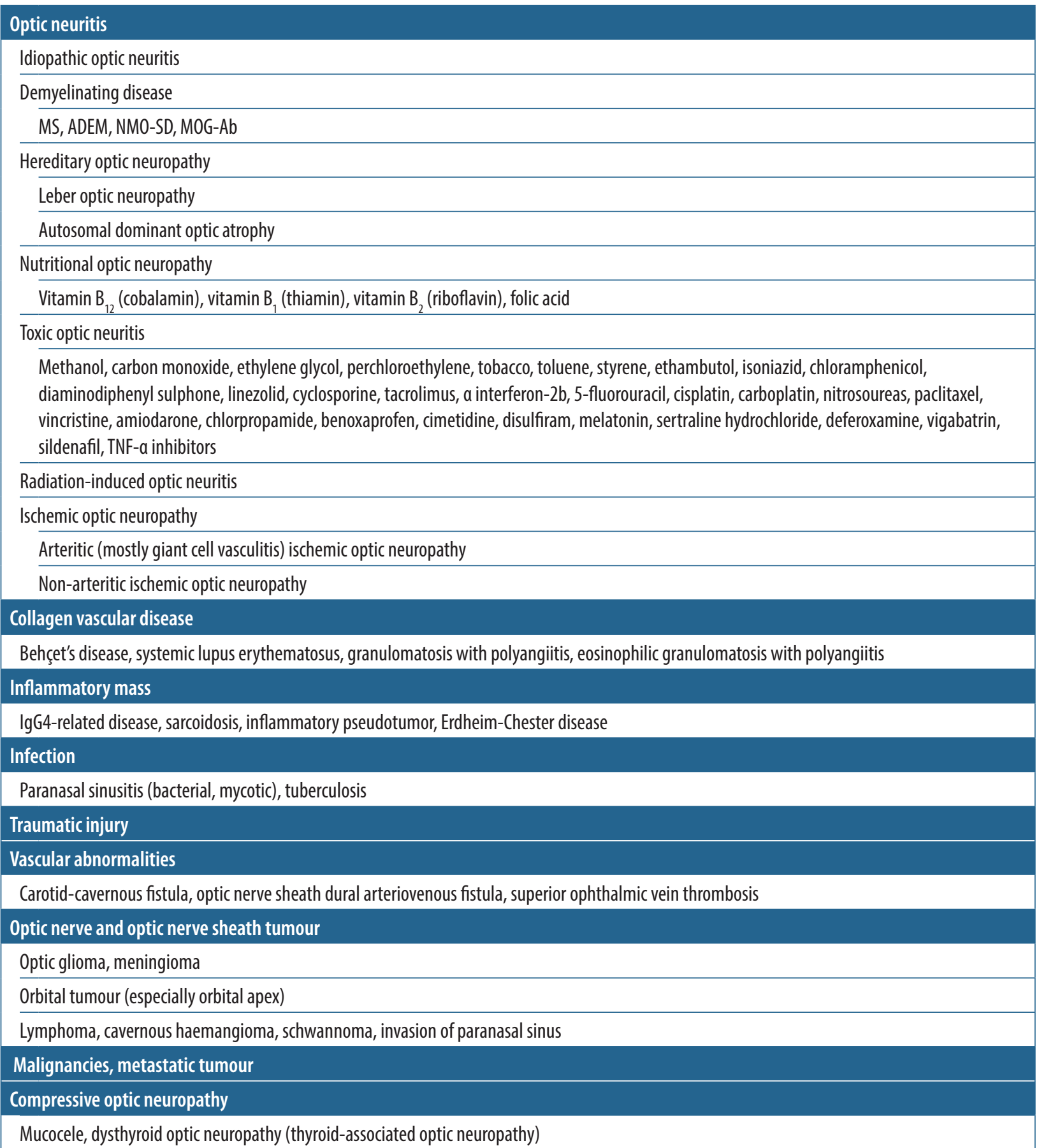

TNF- $\alpha$ - antitumour necrosis factor $\alpha$, MS - multiple sclerosis, ADEM - acute disseminated encephalomyelitis, NMO-SD - neuromyelitis optica spectrum disorder, MOG-Ab - myelin oligodendrocyte glycoprotein antibody, lgG4 - immunoglobulin G4

post-contrast fat-suppressed T1-weighted images are common clinical MRI protocols for the optic nerve. MRI magnetic field strength of $1.5 \mathrm{~T}$ or $3 \mathrm{~T}$ is recommended, and the slice thickness should be less than $3 \mathrm{~mm}$. The orbit and cavernous sinus should be included in both axial and coronal images [2].

\section{Idiopathic optic neuritis}

Idiopathic optic neuritis is an inflammation of the optic nerve of unknown cause. Idiopathic optic neuritis com- monly affects women in their 20s or 30s. Typically, patients experience acute unilateral optic neuritis, resulting in vision loss and pain. Diagnosis of idiopathic optic neuritis requires the exclusion of demyelinating diseases such as multiple sclerosis (MS), neuromyelitis optica spectrum disorders (NMO-SD), and myelin oligodendrocyte glycoprotein antibody (MOG-Ab)-associated optic neuropathy [3]. Even if a diagnosis of idiopathic optic neuritis is made, patients often develop MS, especially in cases in which abnormalities are detected on brain MRI. In patients with optic neuritis, orbital MRI for the evaluation of 

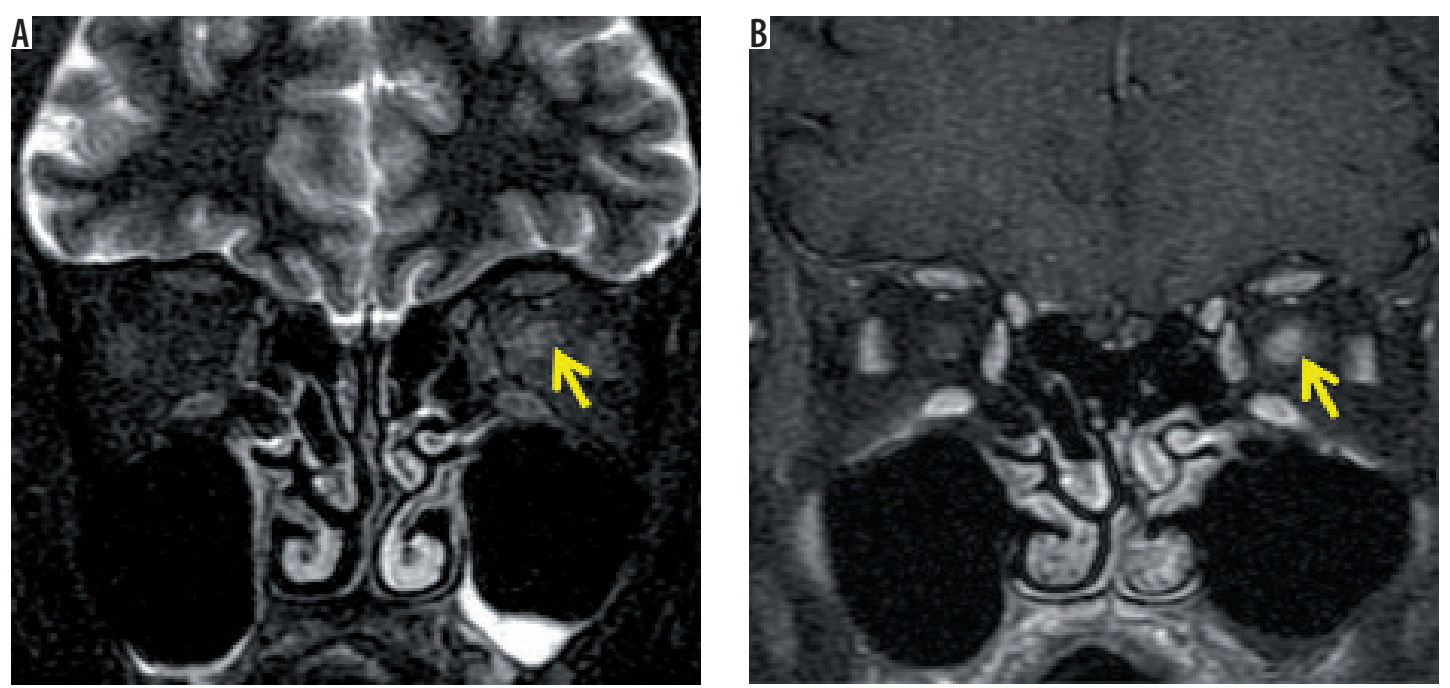

Figure 1. Idiopathic optic neuritis. The left optic nerve has high signal intensity on the STIR image (A) and is enhanced on the fat-suppressed contrast T1-weighted image (B)

optic neuritis and brain MRI for the negation of demyelinating disease are usually performed [4]. Idiopathic optic neuritis is evaluated by MRI, but the findings are non-specific. In the acute phase, the optic nerve is enlarged with high-intensity signal on T2-weighted or STIR images and has a contrast effect on post-contrast-enhanced T1-weighted image (Figure 1). On diffusion-weighted images, the optic nerve shows high signal with decreasing $\mathrm{ADC}$ value. In the chronic phase, the optic nerve is atrophied, with high-intensity signal on T2-weighted images, low-intensity signal on diffusion-weighted images, and poor contrast effect on post-contrast images [5].

\section{Demyelinating disease}

Acute disseminated encephalomyelitis (ADEM), MS, and NMO-SD are known inflammatory demyelinating diseases that cause optic neuritis. These disease names are heterogeneous in nature and the diseases are best viewed as 'syndromes' rather than specific disorders. In recent years, the detection of aquaporin-4 antibodies (AQP4-Ab) and MOG-Ab, which are the causative antibodies of inflammatory demyelinating disease, has been used for diagnosis, and the diagnosis of inflammatory demyelinating diseases is changing drastically [6-9].

ADEM is an inflammatory demyelinating disease that is common in children and is thought to be an immune system dysregulation triggered by an infection or vaccine administration. Preinfection exists in $72-77 \%$ of patients with ADEM about one week before onset, and ADEM develops with symptoms such as: fever, headache, vomiting, visual abnormality, consciousness disturbance, and encephalitis. ADEM is usually monophasic, but when it recurs, it is classified as polymorphic ADEM, MS, or NMO-SD [9]. On brain MRI, a high- signal area on T2-weighted images is primarily observed in the subcortical white matter and is partially accompanied by a contrast effect. An abnormal signal is also found on the optic nerve, but it is indistinguishable from other types of optic neuritis (Figure 2) [10].

Multiple sclerosis is a disease that causes demyelination in the brain, spinal cord, and optic nerve, and it repeats cycles of relapse and remission during its course. MS often accompanies optic neuritis and is an important differential diagnosis from optic neuritis. MS is prevalent among women in their 20s to 30s and, unlike ADEM, it rarely develops before puberty [11]. MS is diagnosed by clinical and MRI findings according to the McDonald criteria, but the image findings of optic neuritis are not included in the McDonald criteria. Optic nerve lesions in patients with MS show a high signal on T2-weighted images and have a contrast effect, but the lesions cannot be distinguished from other types of optic neuritis. In brain MRI, a high-signal area is observed in T2-weighted images, mainly around the periventricular white matter. Patients who develop optic neuritis should undergo brain MRI to exclude MS [12].

Neuromyelitis optica spectrum disorders, which are characterised by a positive test for AQP4-Ab, forms demyelinating lesions in the optic nerve and spinal cord. Previously, NMO-SD was regarded as a subtype of MS, but it was reclassified as an independent disease due to the discovery of AQP4-Ab. NMO-SD typically develops in women in their late 30s, with symptoms of transverse myelitis and bilateral optic neuritis. Diagnosis of NMO-SD is based mainly on AQP4-Ab, MRI, and clinical findings [8]. The optic nerve lesion in NMO-SD, like MS, is nonspecific on MRI (Figure 3), but exists for longer than that in MS [13]. On brain MRI, high signal on T2-weighted images is seen mainly in the dorsal side of the medulla, the fourth ventricle peripheries, the thalamus, and the hypothalamus, where the expression of AQP4 is high [14].

In recent years, MOG-Ab has also been found to cause demyelination in the central nervous system. Although MOG-Ab was first reported as a cause of AQP4-negative 

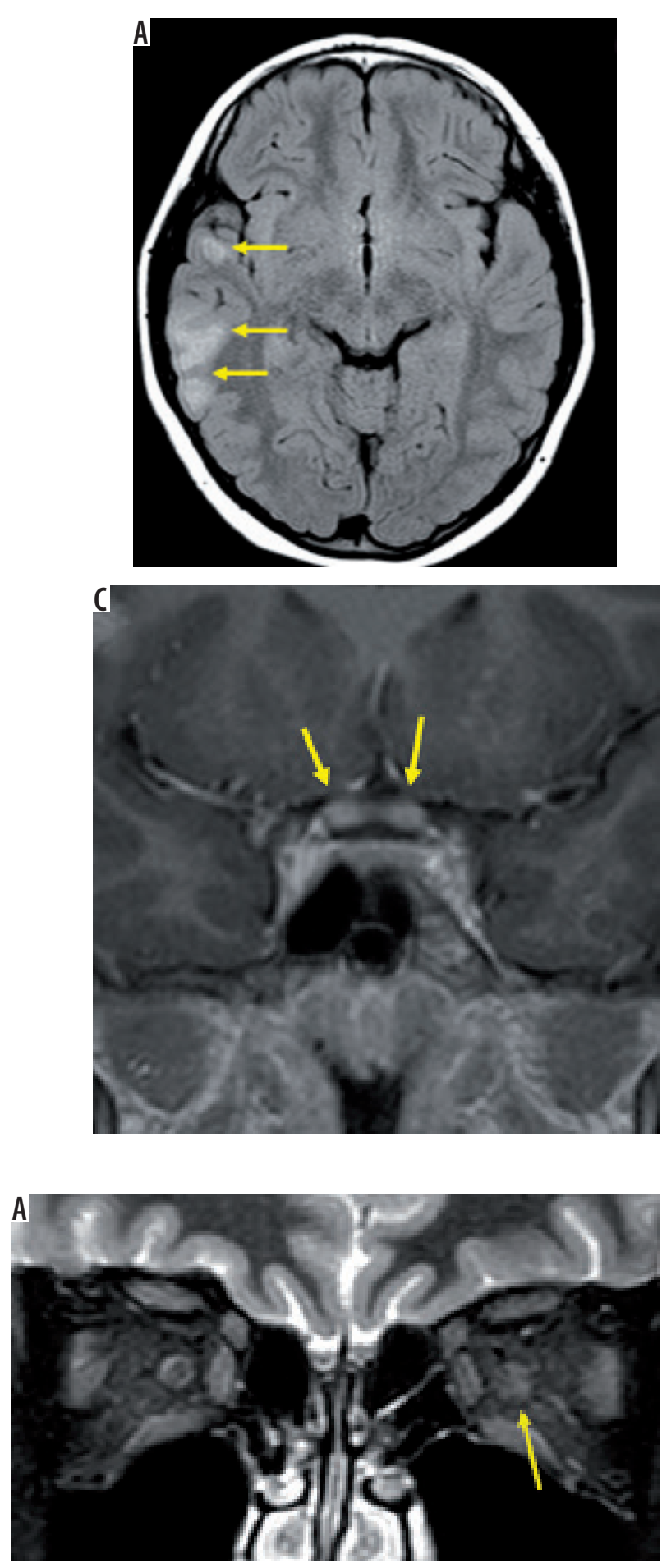

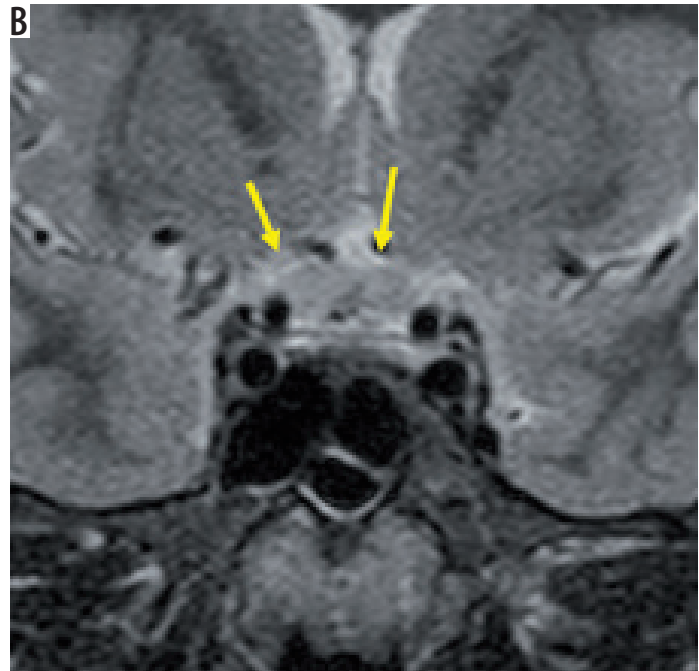

Figure 2. Optic neuritis due to acute disseminated encephalomyelitis. There are high-signal areas in the subcortical white matter on the right in the FLAIR image (A). The chiasm is enlarged with high signal intensity on the T2-weighted image (B). The chiasm is enhanced on the post-contrast T1-weighted image (C)

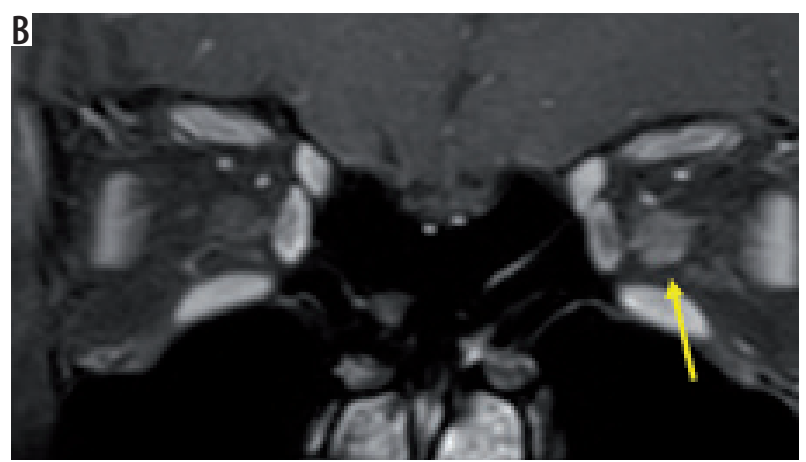

Figure 3. Optic neuritis due to neuromyelitis optica spectrum disorders. The left optic nerve shows a high signal and is swollen on the STIR image (A), and is enhanced on the fat-suppressed contrast T1-weighted image (B)

NMO-SD, MOG-Ab is also detected in patients diagnosed with idiopathic optic neuritis or ADEM [15]. Like other MRI findings in optic neuritis, the optic nerve lesion in MOG$\mathrm{Ab}$ shows high-intensity signal on $\mathrm{T} 2$-weighted images with a contrast effect. The MOG-Ab-positive optic nerve lesion develops in the relatively anterior part of the optic nerve, whereas the AQP4-Ab-positive forms more posteriorly in the optic nerve. A contrast effect spreading around the optic nerve sheath is more suggestive of an MOG-Ab positive optic nerve lesion than an optic nerve lesion due to MS or an AQP4-Ab-positive optic nerve lesion (Figure 4) [16].

\section{Hereditary optic neuropathy}

Leber hereditary optic neuropathy and autosomal dominant optic atrophy are well-known hereditary neuropa- 
thies, but other hereditary optic neuropathies, such as congenital recessive optic atrophy, apparent sex-linked optic atrophy, and autosomal recessive chiasmal optic neuropathy, are all extremely rare [17].

Leber hereditary optic neuropathy is a maternally inherited disorder caused by a mitochondrial abnormality. Leber hereditary optic neuropathy typically affects men, and patients usually develop subacute, progressive vision loss in their teens or 20s. Initially, the loss of vision is unilateral, but after several months vision in the contralateral eye also decreases. Unlike other types of optic neuritis, Leber hereditary optic neuropathy in its acute phase does not cause any abnormality on orbital MRI. However, in the chronic phase, the posterior optic nerve and optic chiasm enlarge with hyperintense signal on T2-weighted images [18].

\section{Nutritional optic neuropathy}

Nutritional optic neuropathy is a condition that causes bilateral loss of visual acuity due to nutrient deficiency. Nutritional optic neuropathy is rarely present in developed countries but may develop in individuals with extreme weight loss or an extremely unbalanced diet. Deficiency of vitamin $B_{12}$ (cobalamin), vitamin $B_{1}$ (thiamin), vitamin $B_{2}$ (riboflavin), or folic acid can cause nutritional optic neuritis. Nutritional optic neuropathy is diagnosed based on a lack of nutrients in the blood. There are a few reports that MRI is useful in diagnosing nutritional optic neuropathy [19]. In patients with vitamin B1 deficiency (Wernicke's encephalopathy), a high-signal area appears in the cerebral aqueduct, inside the thalamus, midbrain, and mammillary body, so brain MRI may be useful for diagnosis [20].

\section{Toxic optic neuritis}

Toxic optic neuritis is a neuropathy caused by the ingestion of toxic substances or by side effects of drugs. Numer-

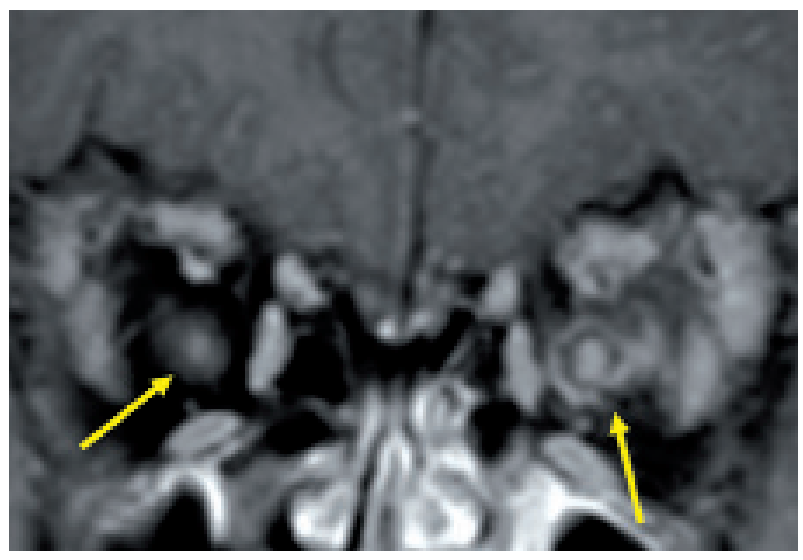

Figure 4. Myelin oligodendrocyte glycoprotein antibody-positive optic neuropathy. Bilateral optic nerves and fat tissue around the left optic nerve sheath (yellow arrow) are enhanced on the post-contrast, fat-suppressed, T1-weighted image

ous toxins and drugs are associated with optic neuritis. The mechanisms of optic nerve toxicity vary depending on the agent. Bilateral loss of vision is a typical symptom, and other neurological complaints may be present in some cases. Since most image findings of toxic optic neuritis are nonspecific, like other forms of optic neuritis, or are unknown, orbital MRI is performed to exclude other diseases that cause optic neuritis $[21,22]$.

\section{Radiation-induced optic neuritis}

Radiation-induced optic neuritis is an essential complication of radiation therapy involving the anterior visual pathway; this neuritis can occur with a total dose of $\geq 50$ Gy or a single dose of $\geq 10$ Gy. Radiation-induced optic neuritis typically causes sudden unilateral vision impairment at around one year after radiotherapy, but occasionally occurs after three years. Within months after vision loss, the optic nerve in the irradiated area displays iso- or hyper-
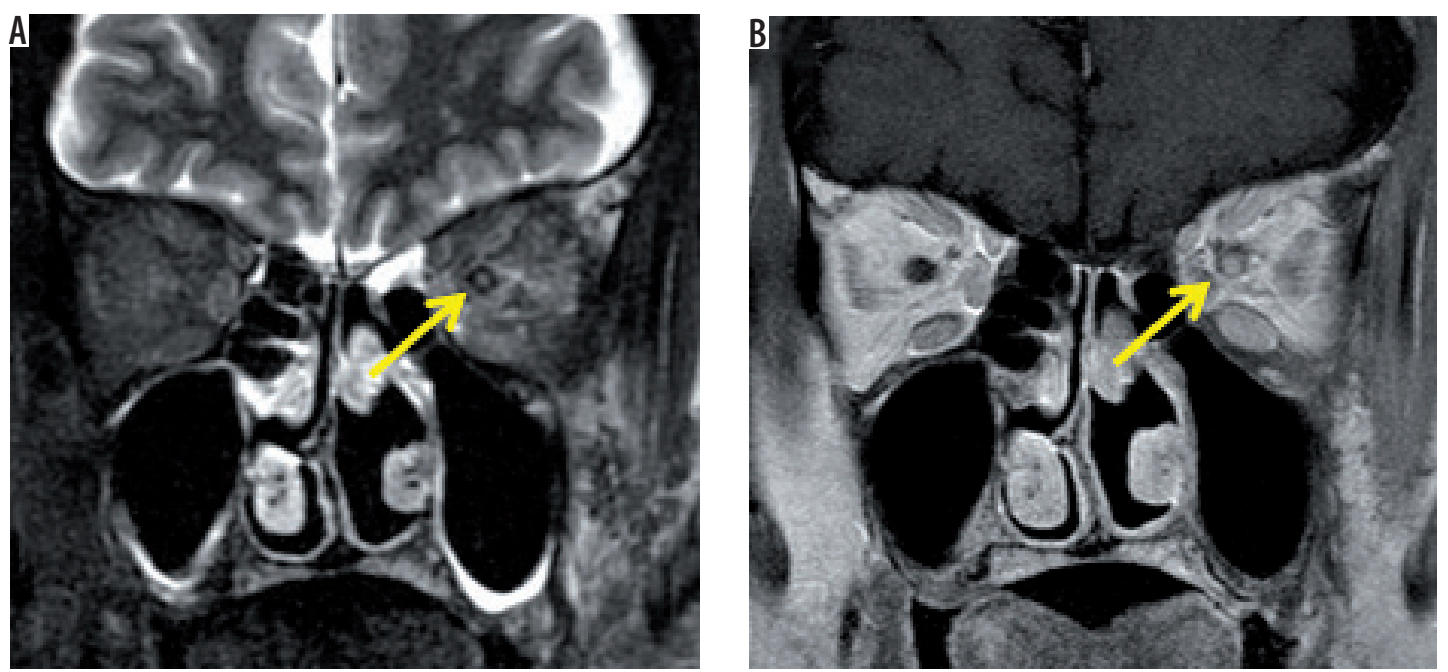

Figure 5. Radiation-induced optic neuritis. There is faint high signal intensity in the optic nerve, and the optic nerve sheath signal is lower than the surrounding tissue on the STIR image (A). The optic nerve is enhanced on the post-contrast, T1-weighted image (B) 
intensity on T2-weighted images, and contrast effect on T1-weighted images (Figure 5), but the contrast effect disappears within a few months [23].

\section{Ischaemic optic neuropathy}

Ischaemic optic neuropathy is the most common cause of unilateral optic neuropathy in patients over age 50 years. Ischaemic optic neuropathy is caused not by inflammation, but by impaired blood flow to the optic nerve. Ischaemic optic neuropathy is classified as either arteritic (mostly giant cell vasculitis) or non-arteritic (in which a shortage of blood flow to the optic nerve is suspected). Non-arteritic ischaemic optic neuropathy results from disease of the small vessels, but its exact cause remains unknown. The most common systemic disorders associated with non-arteritic ischaemic optic neuropathy are hypertension and diabetes mellitus.

Ischaemic optic neuropathy can be further classified into an anterior form, involving the papilla, and a posterior form, which does not involve the papilla. Anterior ischaemic optic neuropathy is diagnosed based on the presence of papilledema, but posterior ischaemic optic neuropathy is usually diagnosed by excluding diagnoses of other kinds of optic neuropathy, because it does not involve papilloedema [24].

MRI is useful for evaluating arteritic ischaemic optic neuropathy due to giant-cell arteritis. The optic neuropathy can be detected with minute contrast effects in the optic nerve by using enhanced black-blood 3D T1-weighted images [25]. In addition, MRI can be effectively used to diagnose temporal arteritis, by visualising wall thickening and contrast effect of the temporal artery [26]. Conversely, MRI is less useful in diagnosing non-arteritic ischaemic optic neuropathy because MRI can scarcely depict the optic nerve abnormalities. The main role of MRI in non-arteritic ischaemic optic neuropathy is to exclude other types of optic neuropathy [27].

\section{Collagen vascular disease}

Several collagen vascular diseases cause vasculitis in blood vessels of the optic nerve, resulting in visual impairment. Giant-cell vasculitis is the most common collagen vascular disease-causing optic neuritis, but Behçet's disease, systemic lupus erythematosus (SLE), granulomatosis with polyangiitis (GPA), and eosinophilic granulomatosis with polyangiitis (EGPA) can also cause optic neuritis [28].

Behcet's disease is a chronic, recurrent systemic inflammatory disease, in which the main symptoms are oral aphthae, vulva ulcers, skin lesions, and retinal choroiditis. In the eye, Behcet's disease typically involves uveitis, but sometimes it affects the optic nerve. In MRI, enlargement of the optic nerve with high-intensity signal on STIR imaging and contrast effect around the optic nerve sheath (the so-called sunflower-like sign) is typical [29].
SLE is a chronic autoimmune disease that can affect multiple organ systems. Optic neuritis develops in about $1 \%$ of SLE patients and is usually accompanied by painful unilateral vision loss. The cause of optic neuritis is the autoimmunity by AQP4-Ab and ischaemia due to vasculitis. MRI shows optic nerve enlargement with contrast effect [30].

GPA is a type of necrotic, granulomatous vasculitis that spreads throughout the body, mainly in the upper respiratory tract and lungs; it is related to the antineutrophil cytoplasmic antibodies. Orbital involvement is common in GPA patients, occurring in approximately $50 \%$ of cases, and causes visual impairment due to intraorbital granuloma formation and scleritis. The intraorbital mass of GPA shows low signal intensity on T2-weighted images and is enhanced heterogeneously. GPA often accompanies sinus-wall thickening, and nasal-septal perforation also occurs (Figure 6) [31]. GPA also causes hypertrophic pachymeningitis, but it may progress to the optic nerve sheath and cause optic neuritis. MRI shows contrast effect on the thickened dura and the optic nerve sheath on the affected side, and a high-signal area exists in the optic nerve on T2-weighted images [32].

Eosinophilic granulomatosis with polyangiitis is defined as a systemic, necrotising small-vessel vasculitis, associated with asthma and with blood and tissue eosinophilia. EGPA often develops in the peripheral nerves, but sometimes it progresses to the central nervous system and the optic nerve. Patients with EGPA often suffer from optic neuritis or obstruction of the central retinal artery, resulting in impaired vision. MRI shows high-signal intensity in the orbit on STIR images in EGPA patients (Figure 7) [33].

\section{Inflammatory disease}

A variety of inflammatory or inflammation-like tumour masses can form in the orbit, all of which cause a decrease in visual acuity. Such known tumour masses include immunoglobulin G4 (IgG4)-related disease, sarcoidosis, Erdheim-Chester disease, and inflammatory pseudotumour.

IgG4-related diseases are chronic inflammatory diseases in which blood IgG4 is elevated, forming a tumour that causes IgG4-positive plasma cells to infiltrate various organs. IgG4-related diseases are diagnosed according to imaging findings, elevated blood IgG4, and pathological findings. The orbital MRI findings in IgG4-related diseases are swelling of the lacrimal gland, intraorbital mass, enlargement of the extraocular muscle(s), and enlargement of cranial nerves, such as the suborbital nerve. Hypertrophic meningitis sometimes affects patients with IgG4 diseases, and it can spread to the optic nerve sheath (Figure 8). When IgG4-related hypertrophic meningitis in the optic nerve sheath presses on the optic nerve, visual function can be reduced [34]. 

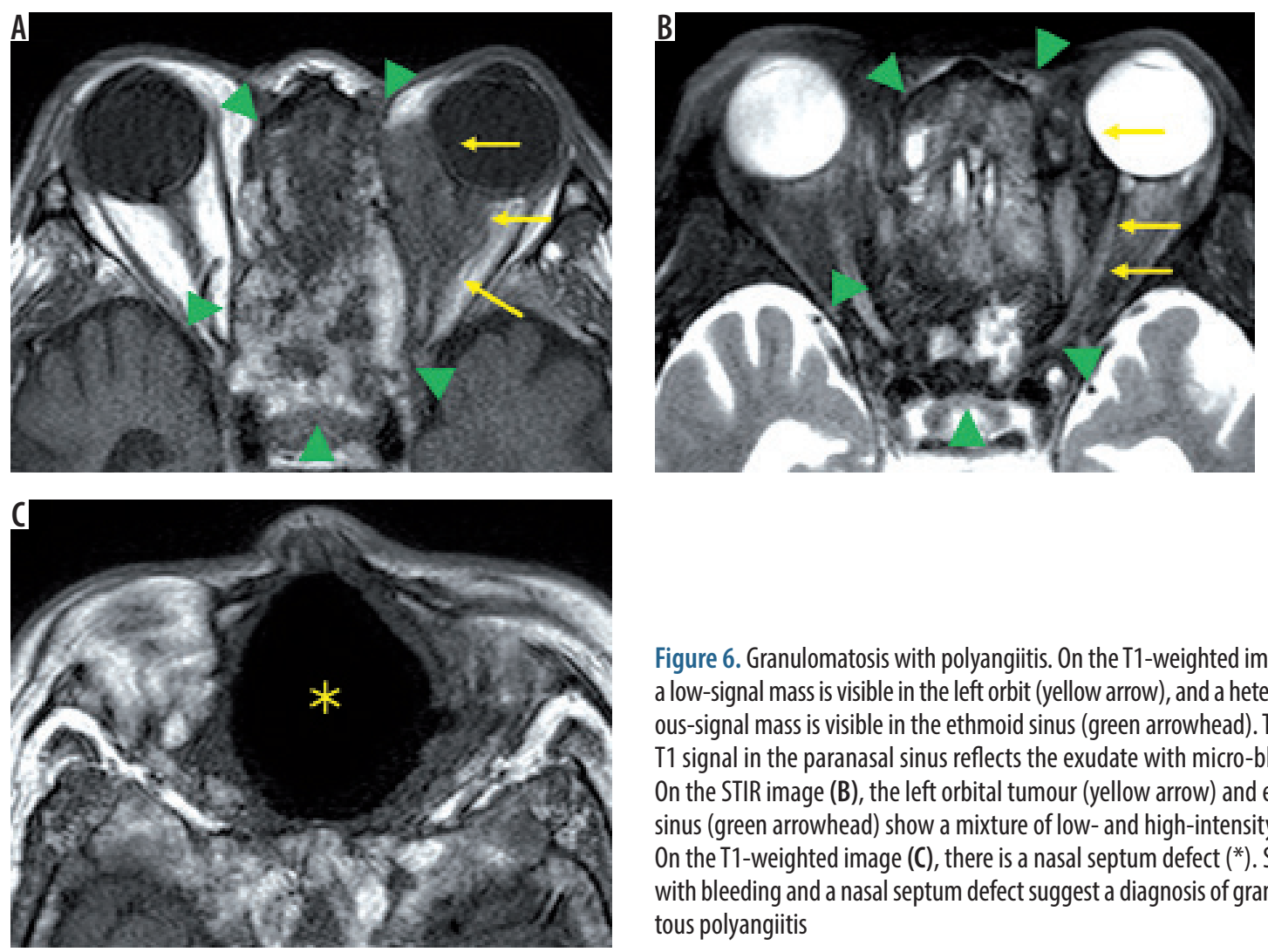

Figure 6. Granulomatosis with polyangiitis. On the T1-weighted image (A), a low-signal mass is visible in the left orbit (yellow arrow), and a heterogeneous-signal mass is visible in the ethmoid sinus (green arrowhead). The high T1 signal in the paranasal sinus reflects the exudate with micro-bleeding. On the STIR image (B), the left orbital tumour (yellow arrow) and ethmoid sinus (green arrowhead) show a mixture of low- and high-intensity signal. On the T1-weighted image (C), there is a nasal septum defect $\left(^{*}\right)$. Sinusitis with bleeding and a nasal septum defect suggest a diagnosis of granulomatous polyangiitis
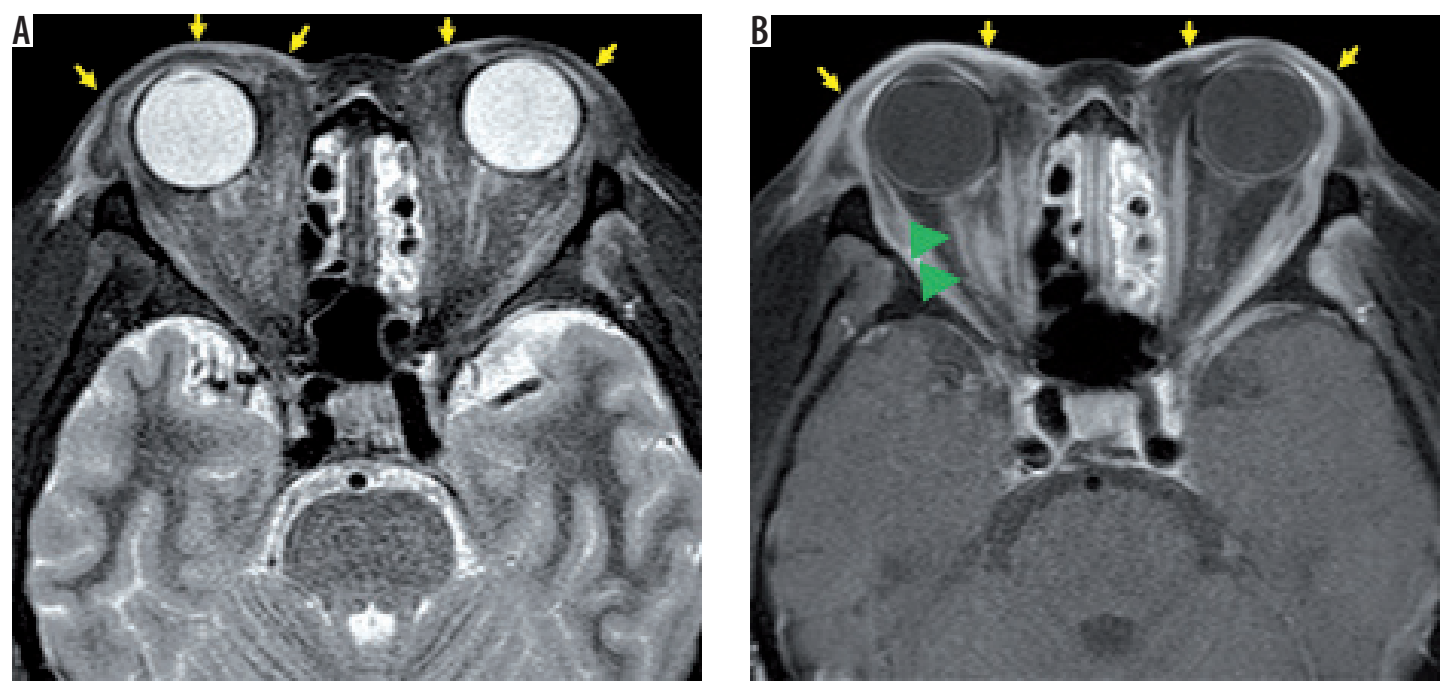

Figure 7. Eosinophilic granulomatosis with polyangiitis. A high signal is observed on both eyelids (yellow arrow) and orbits on the STIR image (A), and there is heterogeneous contrast effect in the eyelids (yellow arrow) and orbits (green arrowheads) on fat-suppressed contrast T1-weighted image (B)

Sarcoidosis is an inflammatory disease of unknown cause, which occurs in multiple organs. Uveitis and retinitis are frequent manifestation of sarcoidosis, but optic nerve lesions are uncommon. However, in cases of neurosarcoidosis, cranial nerve involvement is the most common presenting feature (55\%), with the optic nerve being most commonly affected (33-75\%). Optic nerve sarcoidosis usually occurs unilaterally, but it develops bilaterally in $31 \%$ of patients. With orbital MRI, the optic nerve shows a high signal on T2-weighted images, and contrast effect exists in the optic nerve and optic nerve sheath [35].

Erdheim-Chester disease is a rare non-Langerhans cell histiocytosis that is characterised by xanthomatous infiltration of tissues. Patients with Erdheim-Chester disease usually develop limb-bone pain in their 40s or 50s. Erdheim-Chester disease is characterised by bilateral and symmetric osteosclerosis of the diaphysis of the long 

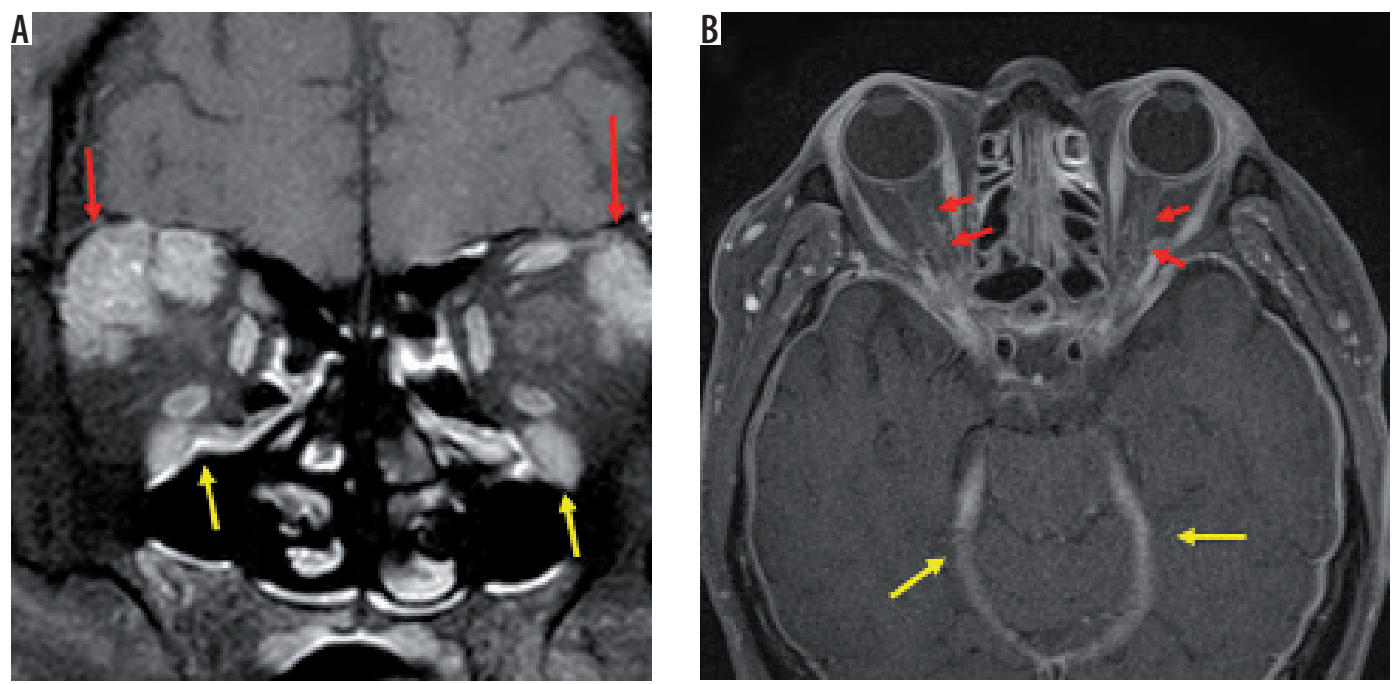

Figure 8. IgG4-related disorders. Two cases of IgG4-related disease are shown. Bilateral lacrimal glands (red arrows) and infraorbital nerves (yellow arrows) are enlarged due to the IgG4-related disease on the contrast-enhanced coronal T1-weighted image (A). The dura (yellow arrows) and the bilateral optic nerve sheaths (red arrows) have been thickened and contrast-enhanced due to the hypertrophic meningitis on the contrast-enhanced, T1-weighted image (B)
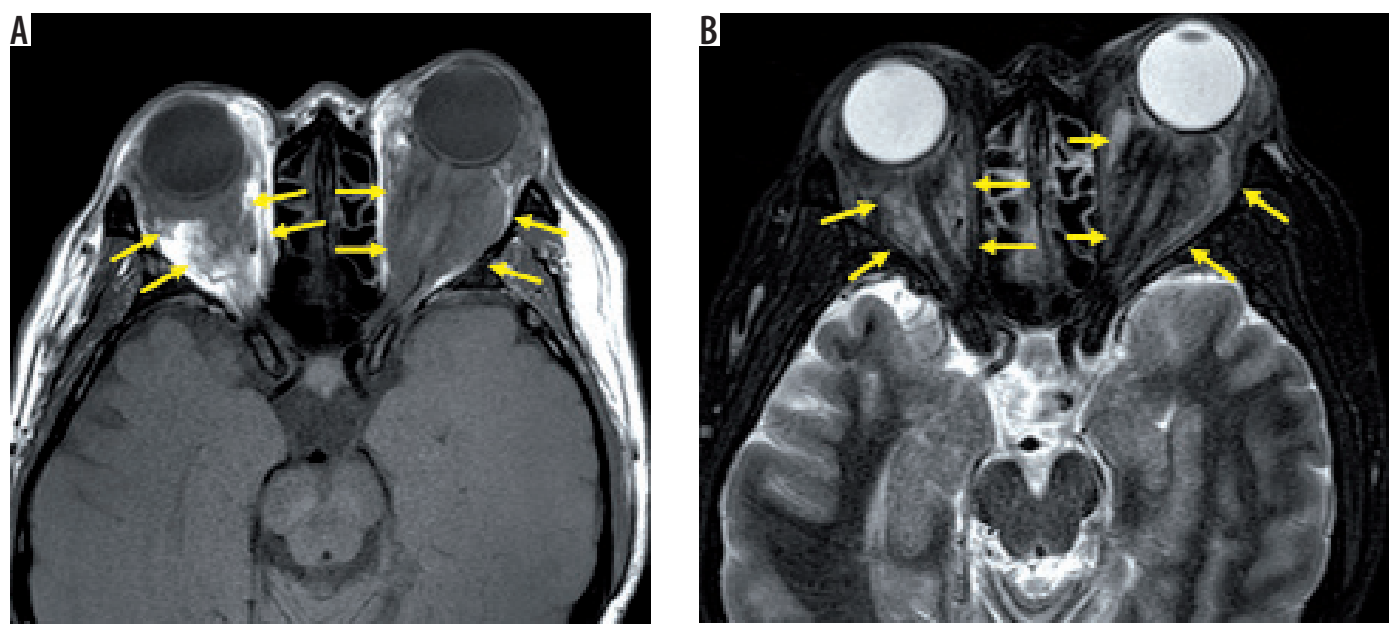

Figure 9. Erdheim-Chester disease. There is a low-signal-intensity mass in the bilateral orbital fat around the optic nerve on the T1-weighted image (A). The mass shows heterogenous signal on the STIR image (B)

bones on X-ray. The orbital lesions mainly show lowintensity signal on both T1- and T2-weighted images, and diffuse enhancement in post-contrast T1-weighted images (Figure 9). Although the mass pressures the optic nerve, vision deterioration is rare [36].

Inflammatory pseudotumour is a non-infectious inflammatory mass, without systemic disease, and is the most common type among painful orbital mass. The cause of inflammatory pseudotumour is unknown, and the diagnosis is made by exclusion, based on clinical and imaging findings. The symptoms differ depending on the lesion site, but when it is close to the optic nerve, it causes a decrease in visual function. Inflammatory pseudotumour occurs around the lacrimal gland, extraocular muscle peripheries, optic nerve sheath circumference, orbital apex, and so on. Inflammatory pseudotumours show low-intensity signal on both T1-and T2-weighted images with orbital MRI (Figure 10) [37].

\section{Infection}

A variety of bacterial infections, fungal infections, and viral infections can occur in the orbit. When inflammation due to infection reaches the optic nerve, visual function deteriorates. Orbital infections usually originate from sinusitis, especially from the ethmoid sinuses, leading to orbital bacterial or fungal infections. Bacterial infections of the orbit are common in healthy individuals. MRIs of patients with bacterial sinusitis-induced optic neuritis show paranasal sinuses and orbital fat with low signals on T1-weighted images; high signals appear on fat-suppression T2-weighted images. The optic nerve is surrounded by the mass and presents a high-intensity signal on T2-weighted images (Figure 11) [38].

Fungal sinusitis is histologically divided into invasive and non-invasive forms. Invasive sinusitis is rare and likely to occur in immunocompromised patients. Fungal optic 

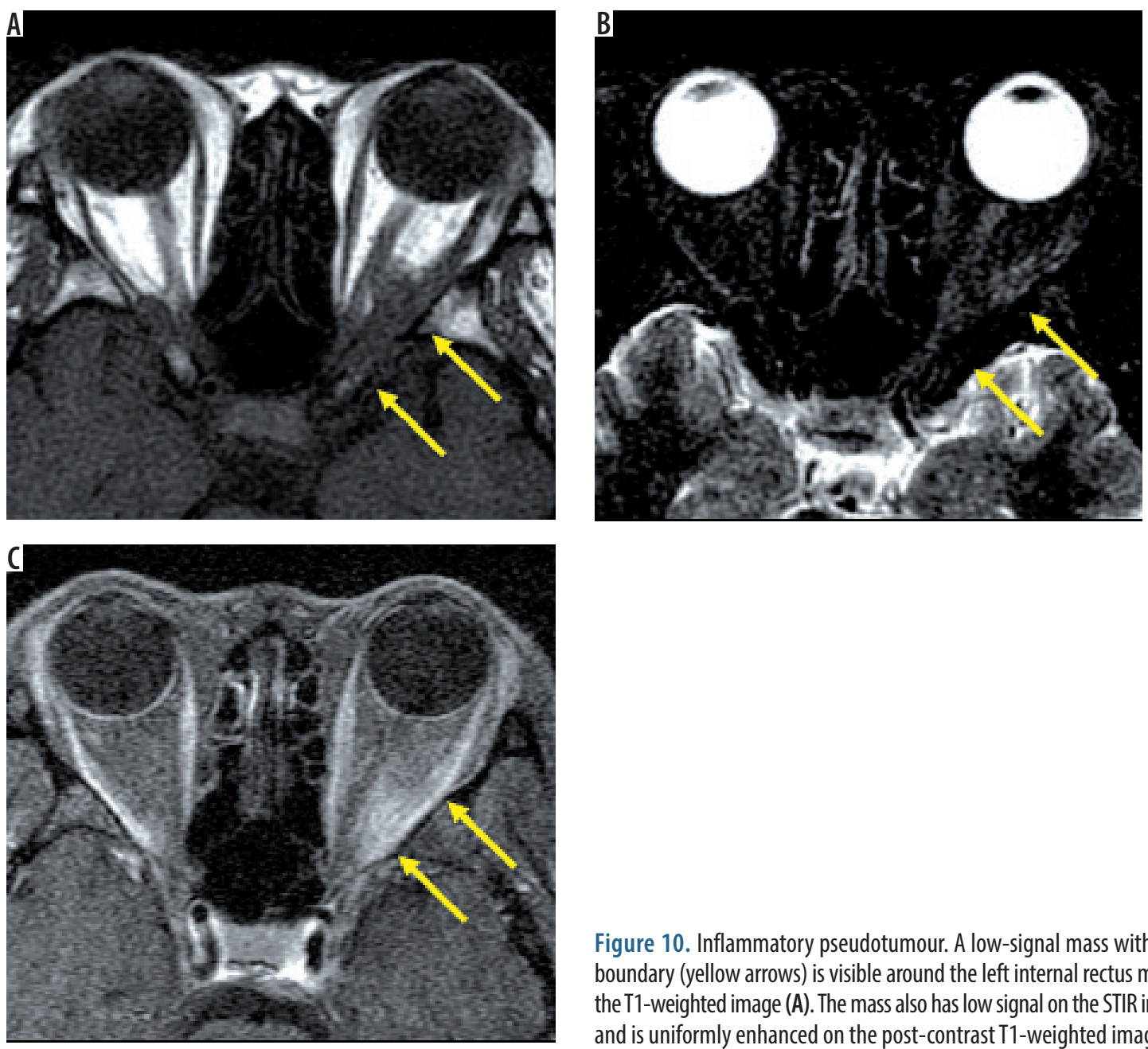

Figure 10. Inflammatory pseudotumour. A low-signal mass with unclear boundary (yellow arrows) is visible around the left internal rectus muscle on the T1-weighted image (A). The mass also has low signal on the STIR image (B) and is uniformly enhanced on the post-contrast T1-weighted image (C)
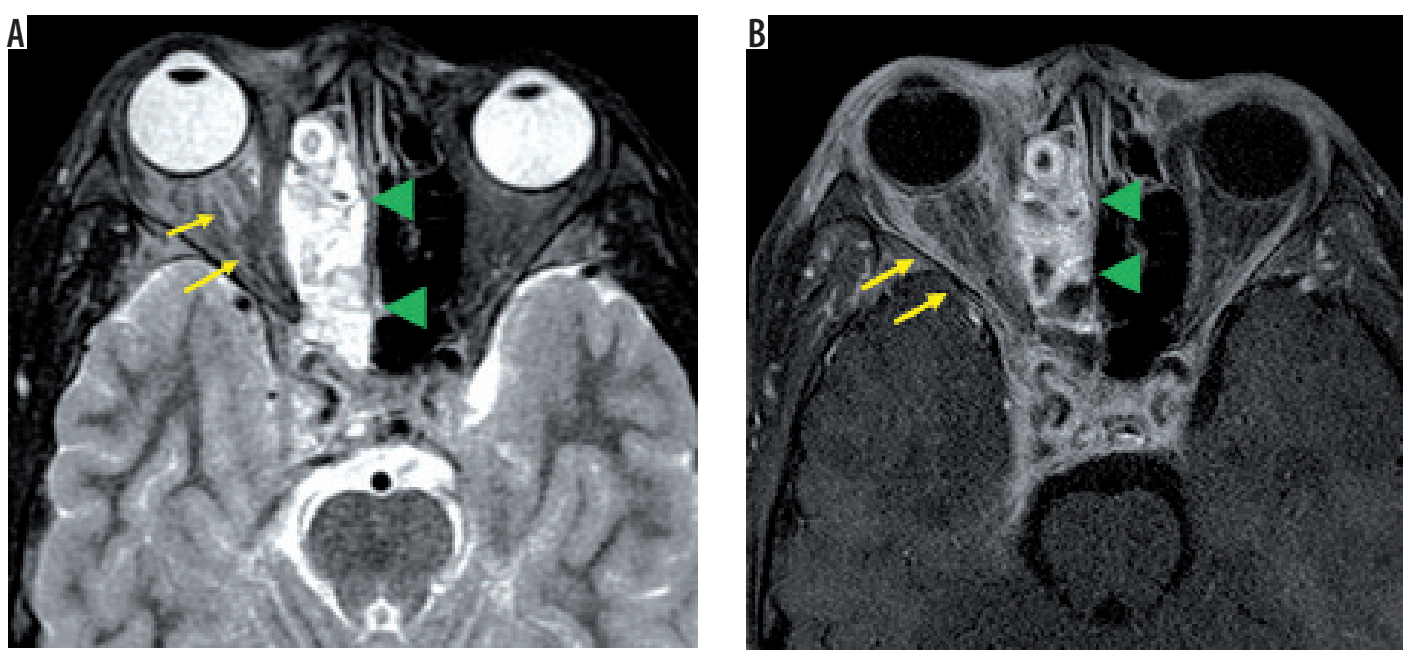

Figure 11. Optic neuritis due to bacterial sinusitis. The mucosa of the right ethmoid sinus is thickened (green arrowheads) with high-intensity signal, and the right orbital fat and the optic nerve (yellow arrow) show high signal intensity. On fat-suppressed contrast T1-weighted image (B), the right ethmoid sinus (green arrowheads) and orbit (yellow arrow) are heterogeneously enhanced

neuritis is caused by invasive sinusitis and is similar to its bacterial counterpart, but sinus-wall thickening and orbital lesions are often limited. Furthermore, fungal infections of the paranasal sinuses and orbits show low signals in T2-weighted images and become obscure in fat-suppressed,
T2-weighted images on MRI. In cases of optic neuritis due to fungal sinusitis, attention should be paid to the abnormality of the fat signal at the orbital apex (Figure 12) [39].

Tuberculous optic neuritis occurs as tuberculous meningitis develops in the optic nerve and optic chiasm. 

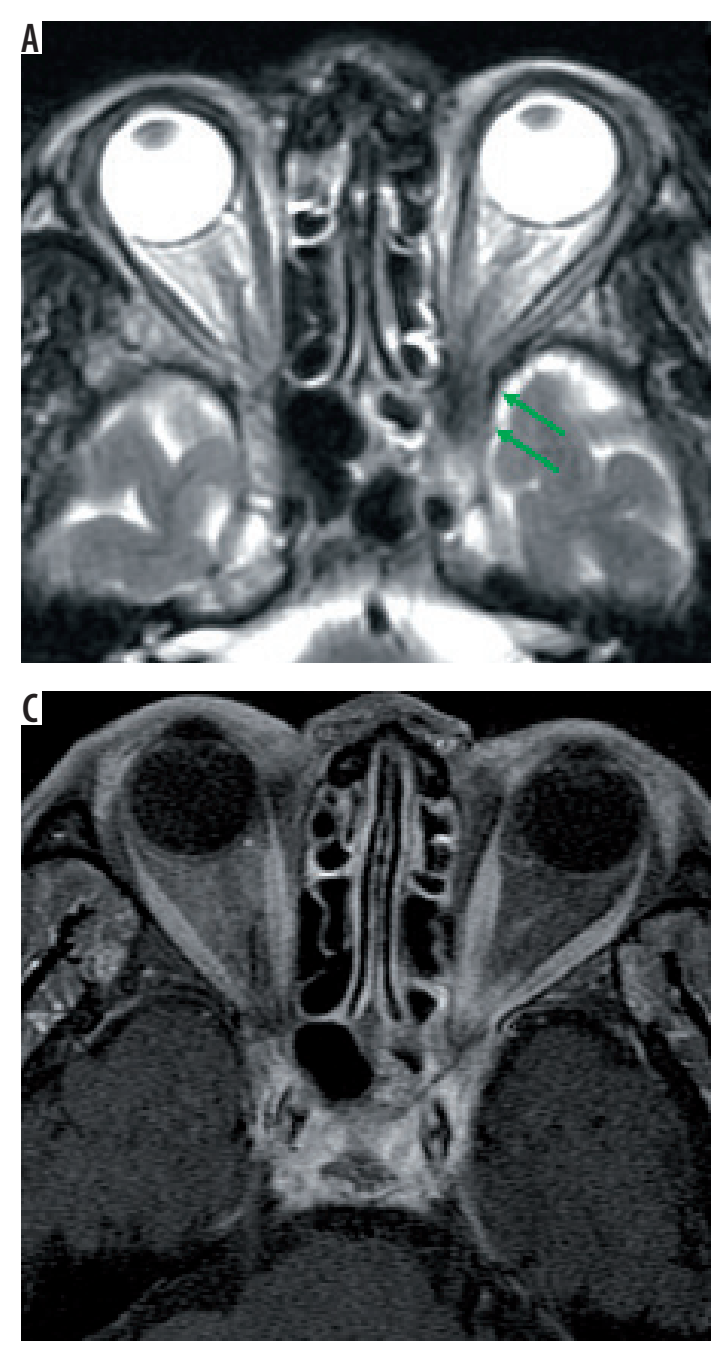

In addition to the presence of meningitis, the ring-shaped contrast effect and swelling are recognisable in the optic nerve in cases of tuberculous optic neuritis. Brain MRI visualises hydrocephalus, meningeal hyperplasia, and tuberculoma, which are findings of tuberculous meningitis [40].

The optic nerve can also be affected by various infectious diseases such as Bartonella, cytomegalovirus, Epstein-Barr virus, human immunodeficiency virus, Lyme disease, syphilis, toxoplasmosis, toxocariasis, and varicella zoster virus, but there are no comprehensive reports about MRI findings in optic neuritis due to these infections [14].

\section{Traumatic optic neuropathy}

Traumatic optic neuropathy is a complication of head and orbit trauma, and can be divided into direct injury and indirect injury cases. Indirect injury is more common than direct injury, and the incidence of traumatic optic neuropathy is estimated to be $0.5-5 \%$ in cases of head trauma. Optic neuropathy due to indirect injury is a traumatic visual disorder with no abnormality in the optic nerve, diagnosed with ophthalmoscopic and image analysis such as CT and MRI. However, in optic neuropathy due to di-

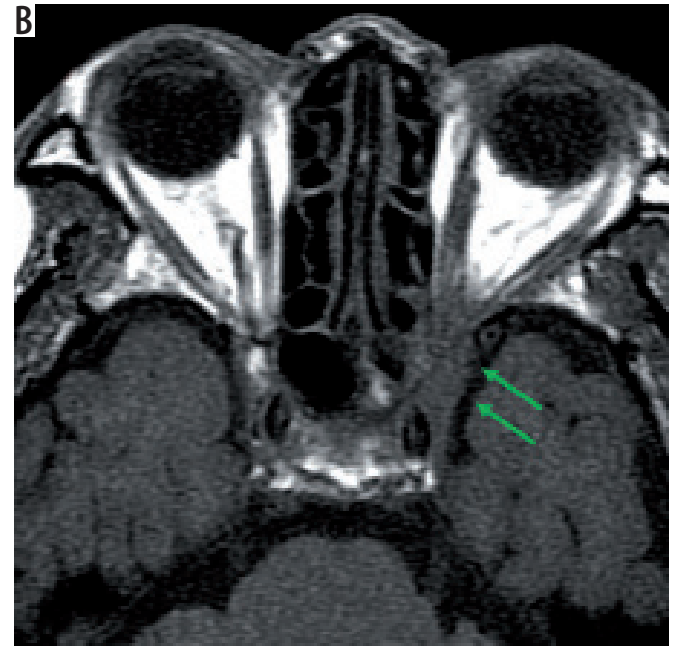

Figure 12. Invasive aspergillosis of the orbital apex. The orbital apex has low signal (green arrows), and the mucosa of the ethmoid sinus is mildly thickened on the STIR image (A). The fat tissue of the orbital apex (green arrows) has low signal intensity compared with the contralateral side on the T1-weighted image (B). It is difficult to detect the lesion on the post-contrast fat-suppressed, T1-weighted image (C)

rect injury, image analysis can visualise bone fragments and foreign bodies that damage the optic nerve. CT is the first test method for traumatic optic neuropathy because it can evaluate the whole body at the same time and can depict most foreign bodies and bone fragments. MRI can visualise optic nerve damage as a high-intensity signal on T2-weighted images, but only plays a small role in diagnosing traumatic optic neuropathy [41].

\section{Vascular abnormalities}

Carotid cavernous fistula (CCF) is an abnormal communication between the carotid artery and vein in the cavernous sinus, due to trauma or the rupture of an aneurysm. Symptoms of CCF include ocular prolapse, red eye, bruit, and double vision, but visual acuity reduction due to high venous pressure also occurs. MRI findings from a CCF show no abnormality in the optic nerve, but they are often accompanied by dilation of the superior ocular vein and cavernous sinus (Figure 13). MR angiography is also used for diagnosis of CCF, but exclusion diagnosis of CCF is difficult when only using MRA, due to poor sensitivity. The diagnosis of CCF is usually based on angiography or CT angiography [42]. 
Superior ophthalmic vein thrombosis is a very rare disorder, which is caused by orbital infections and various kinds of inflammation. The superior ophthalmic vein is responsible for most of the venous perfusion of the orbit, and superior ophthalmic vein thrombosis causes protrusion of the eye, a decrease in visual acuity, and limitations of eye movements. Superior ophthalmic vein thrombus presents an enlarged superior ophthalmic vein with a high-intensity signal in the lumen on T2-weighted images. The vein wall is thickened with a contrast effect, but there is no contrast effect in the lumen (Figure 14). Cavernous sinus thrombosis is often accompanied by superior ophthalmic vein thrombosis [43].

\section{Optic nerve and optic nerve sheath neoplasm}

Of tumours arising from the optic nerve or optic nerve sheath, optic gliomas and meningiomas are common [44]. Optic gliomas are the most common tumours of optic nerve origin. Optic gliomas are common in children under the age of eight years, and most can be classified as juvenile pilocytic astrocytomas. Optic gliomas are often associated with neurofibromatosis type 1 (NF1) and are found in $20 \%$ of NF1 cases. Sporadic optic gliomas are more likely to be localised to the optic chiasm and are more symptomatic than NF1-associated optic gliomas. MRI can visualise the enlargement of the optic nerve and show a high signal on T2-weighted images, but the contrast effect of the tumour varies. There may be a high-signal area at the periphery of the tumour on
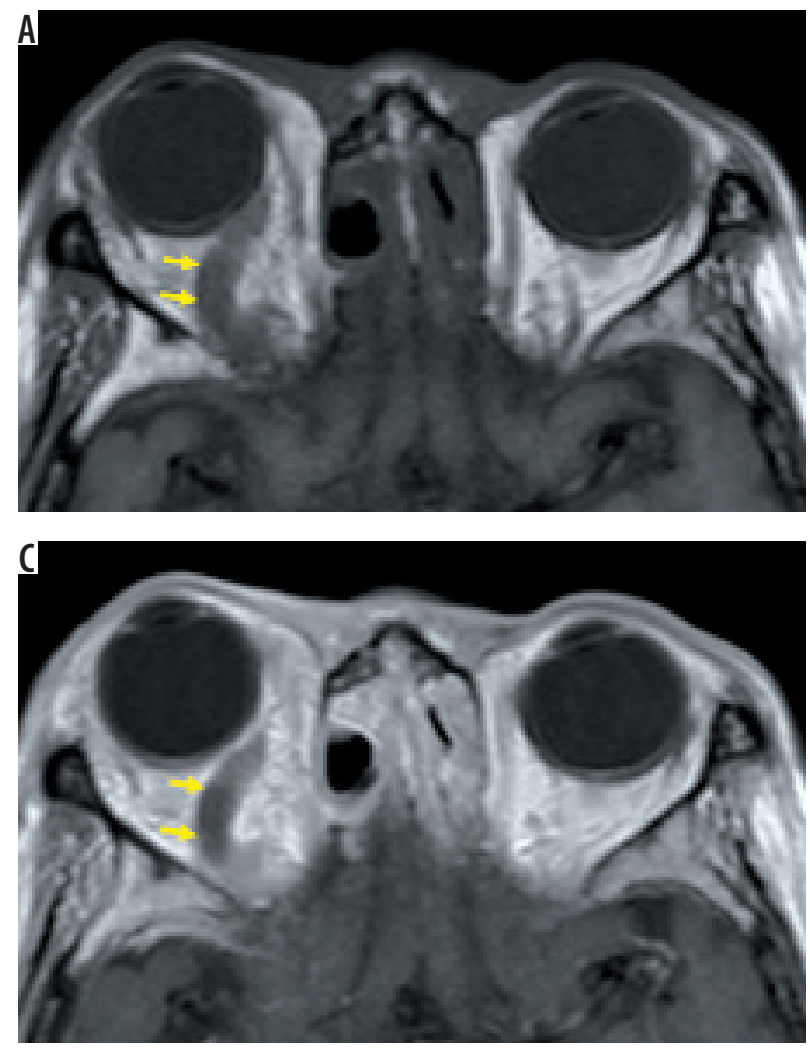

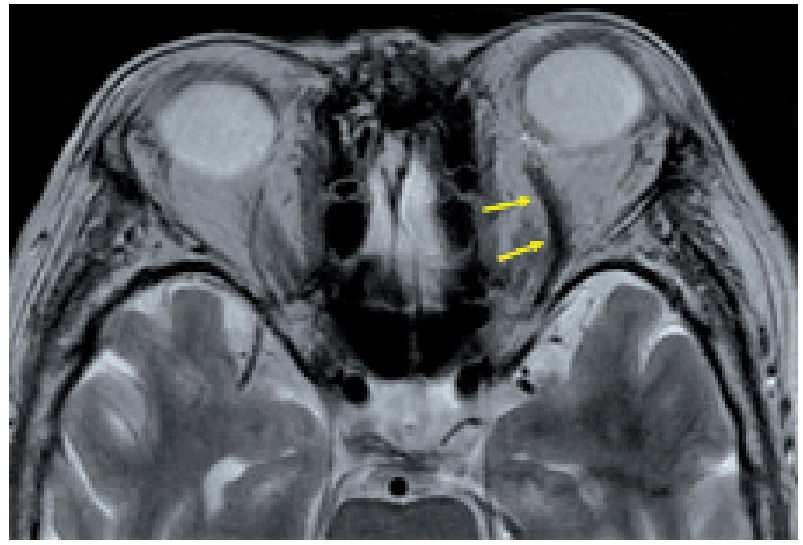

Figure 13. Carotid cavernous fistula. Dilation of the superior ophthalmic vein (arrows) on this T2-weighted image is a finding that casts doubt on the carotid cavernous fistula

T2-weighted images, which is highly suggestive of optic glioma $[44,45]$.

Optic nerve sheath meningioma is a type of meningioma that spreads along the meninges of the optic nerve sheath. Ninety per cent of optic nerve sheath meningiomas develop from the intracranial meninges, with only $10 \%$ originating from the optic nerve sheath. Because the optic nerve sheath is narrow, most optic nerve sheath meningiomas compromise visual function. MRI displays a soft-tissue mass along the optic nerve sheath, and the contrast effect along the optic nerve sheath can be recognised on post-contrast-enhanced, T1-weighted images. The two linear contrast effects along the optic nerve on

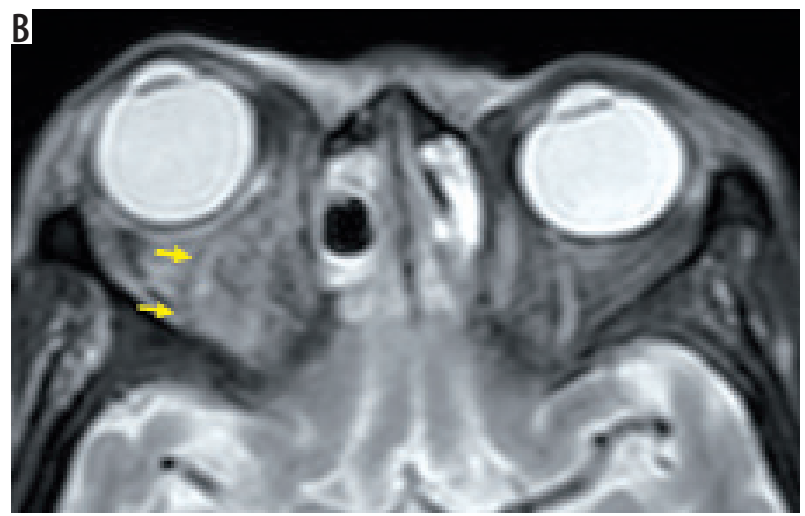

Figure 14. Superior ophthalmic vein thrombus. The right superior ophthalmic vein (yellow arrows) is dilated on the T1-weighted image (A), and the lumen has high signal intensity on the STIR image (B) without contrast effect on the post-contrast, T1-weighted image (C) 


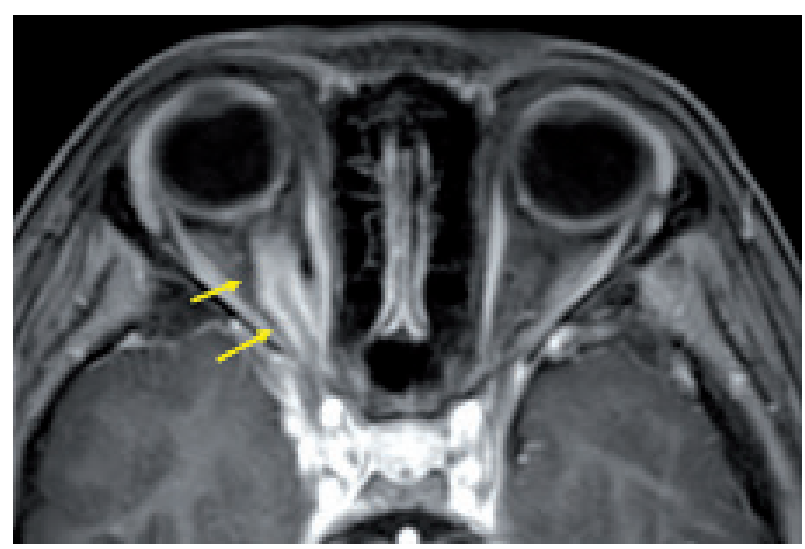

Figure 15. Optic nerve sheath meningioma. The optic nerve sheath is thickened with a contrast effect on this post-contrast T1-weighted image. This double-line contrast effect is the so-called tram-track sign

contrast-enhanced, axial T1-weighted images are called the "tram-track sign" (Figure 15), but this finding is not specific to optic nerve meningiomas because it is also found in cases of optic perineuritis. CT aids in diagnoses by depicting calcification in the tumour [44].

\section{Orbital tumour}

The optic nerve is compressed by various tumours, which results in visual function reduction, particularly
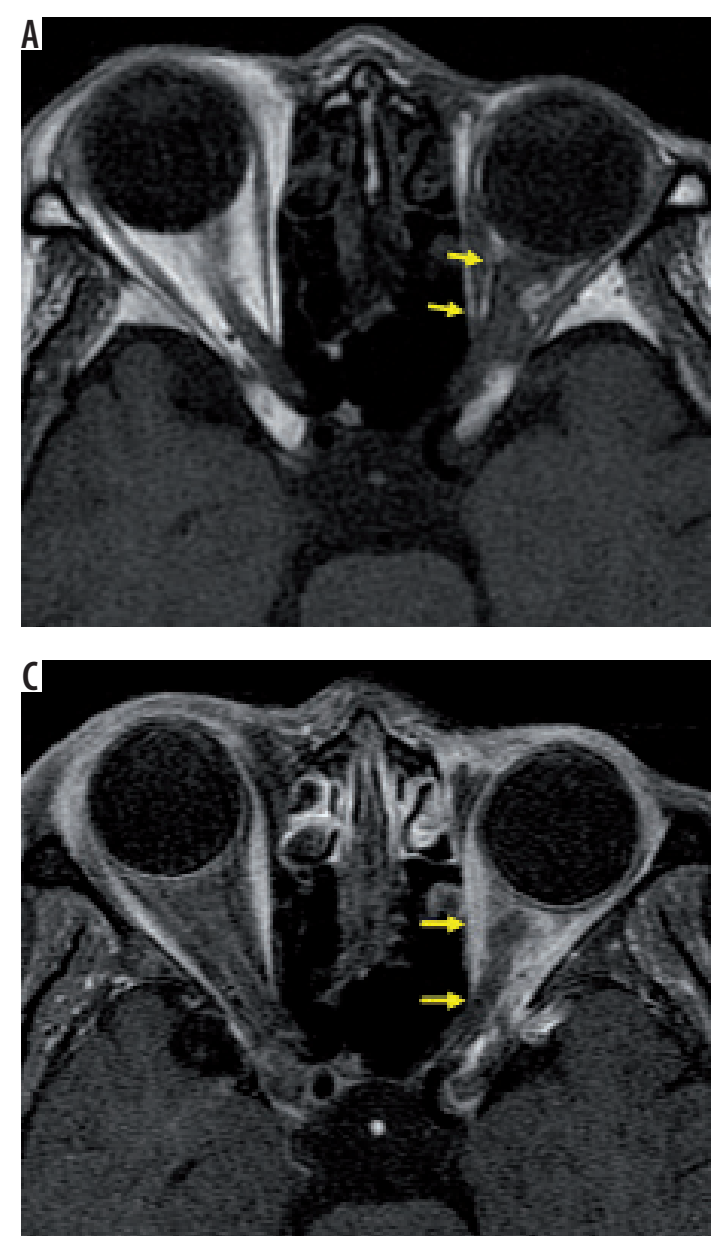

with tumours at the orbital apex. Tumours that compress the optic nerve include primary orbital tumours, direct tumour invasion from an adjacent structure, and metastatic orbital tumours. Of orbital tumours, lymphoma, cavernous haemangiomas, and schwannomas frequently occur, and, even if they are benign, they press on the optic nerve [44].

Lymphoma is the most frequently occurring of intra orbital tumours and is common in people over the age of 60 years. On MRI, lymphoma is often seen as an ill-defined mass, but sometimes it is a smooth, circumscribed mass. Lymphoma may spread along the existing structures, such as the optic nerve, and this finding is characteristic of lymphoma (Figure 16) [46]. Cavernous haemangiomas are not true tumours but are the most common orbital masses in adults. On MRI, cavernous haemangioma presents a smooth border with hyperintensity on T2-weighted images. Visual abnormalities due to cavernous haemangiomas are rare, but they can cause compressive optic neuritis at the orbital apex. Schwannomas are rare in the orbit, but they arise from the trigeminal, oculomotor, trochlear, and abducens nerves. On MRI, a schwannoma is a well-defined mass that develops along the nerve. A schwannoma is a mixture of components of Antoni A areas, characterised by rich cellular areas, and Antoni B areas, characterised by poor cellular areas, and it exhibits a complicated sig-

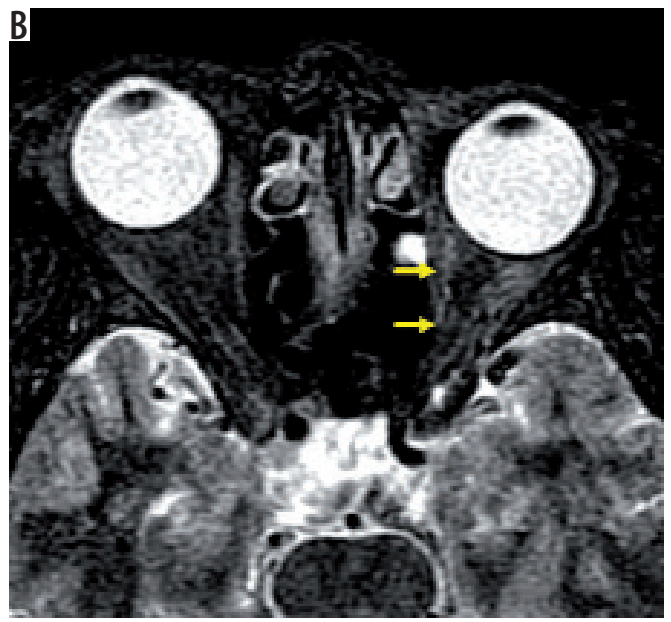

Figure 16. Lymphoma. An ill-defined low-signal mass (yellow arrows) is located around the left optic nerve on the T1-weighted image (A). The mass has low signal on the STIR image (B) and contrast effect on the post-contrast, fat-suppressed, T1-weighted image (C) 

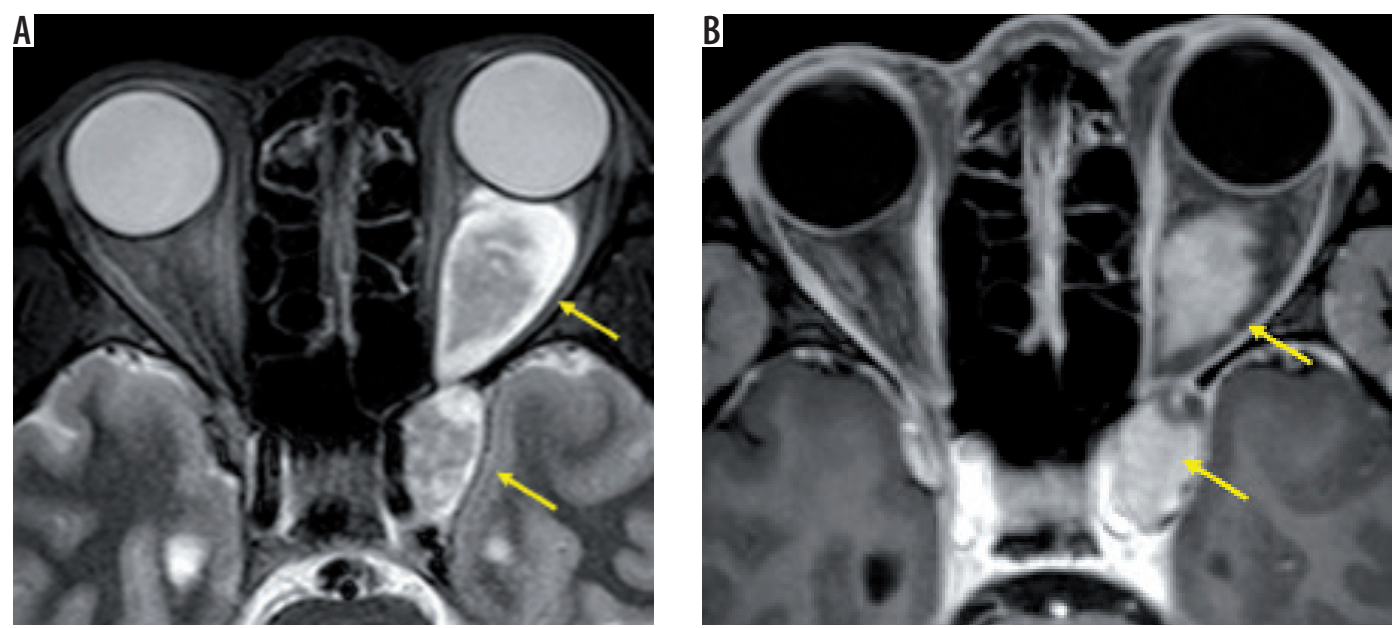

Figure 17. Schwannomas of the oculomotor nerve. A dumbbell-shaped tumour exists from the cavernous sinus to the orbit (yellow arrows). The tumour periphery has high signal intensity, and the interior is iso-intense with the brain on the STIR image (A). The inside of the tumour is enhanced on the post-contrast, fat-suppressed, T1-weighted image (B)

nal on T2-weighted images (Figure 17). Although visual performance rarely deteriorates, it can if the optic nerve is compressed [44].

Paranasal malignancies are commonly accompanied orbital invasion. In many cases, the infiltration of the sinus by malignant tumours remains in the orbital periosteum, and it is rare that an optic nerve injury occurs at the beginning. However, local recurrence of the tumour often invades the optic nerve [47].

Metastatic tumours account for $1-13 \%$ of orbital tumours, and $16-23 \%$ of patients develop vision loss. Breast cancer, lung cancer, and prostate cancer are common sources of orbital metastases, but their frequency differs depending on the demographics and country of origin of the study. The image findings of metastatic tumours are nonspecific except for forming mass in the orbit (Figure 18), and the history of malignant tumours is important [48].

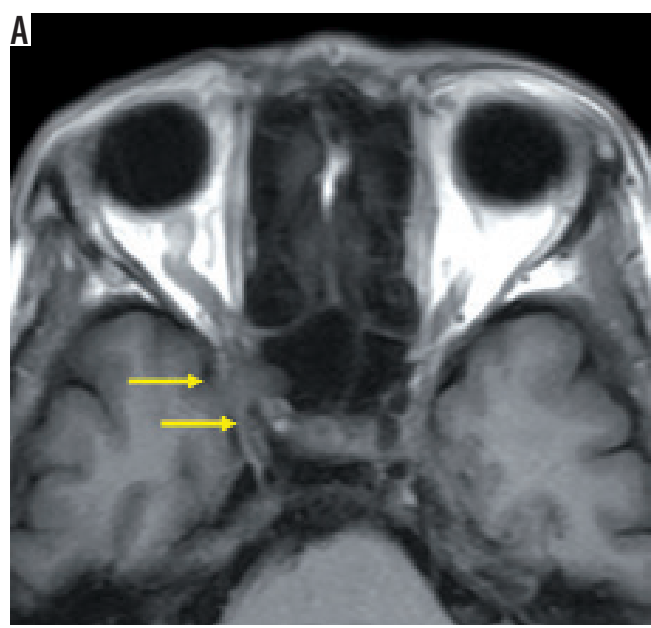

\section{Compressive optic neuropathy}

Compressive optic neuritis is a form of neuritis that occurs when the optic nerve is compressed by a mass. This section deals with compression that is not caused by tumours or inflammatory masses.

A mucocele is not a tumour, but it forms encapsulated mucous cavities in the paranasal sinuses. As the mucocele increases, it progresses to the orbit and compresses the optic nerve. When the mucocele occurs in the Onodi cell surrounding the optic nerve, or the anterior floor processes, it is close to the optic nerve and often causes acute vision loss. A mucocele is a smooth-margin mass that causes bulging in the surrounding bone, and in many cases it is easy to distinguish from malignant paranasal sinus tumours. On MRI, a mucocele presents high-intensity signals in both T1-weighted images and T2-weighted images without con-

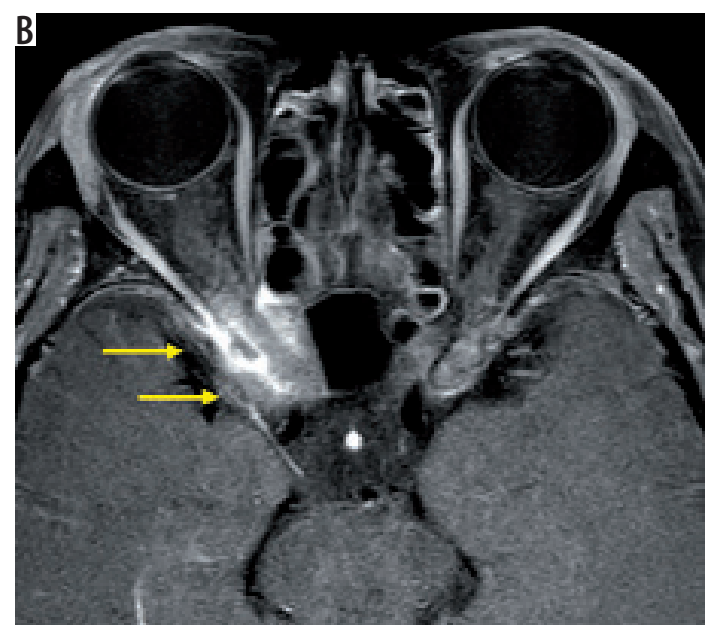

Figure 18. Optic canal metastasis from breast cancer. The right orbital apex has low signal (yellow arrows) compared with the contralateral side on the T1-weighted image (A). The right orbital apex is heterogeneously enhanced (yellow arrows) compared with the contralateral side on the fat-suppressed, post-contrast, T1-weighted image (B). As a result of the whole-body assessment, the tumour was diagnosed as an optic canal metastasis from the patient's breast cancer 

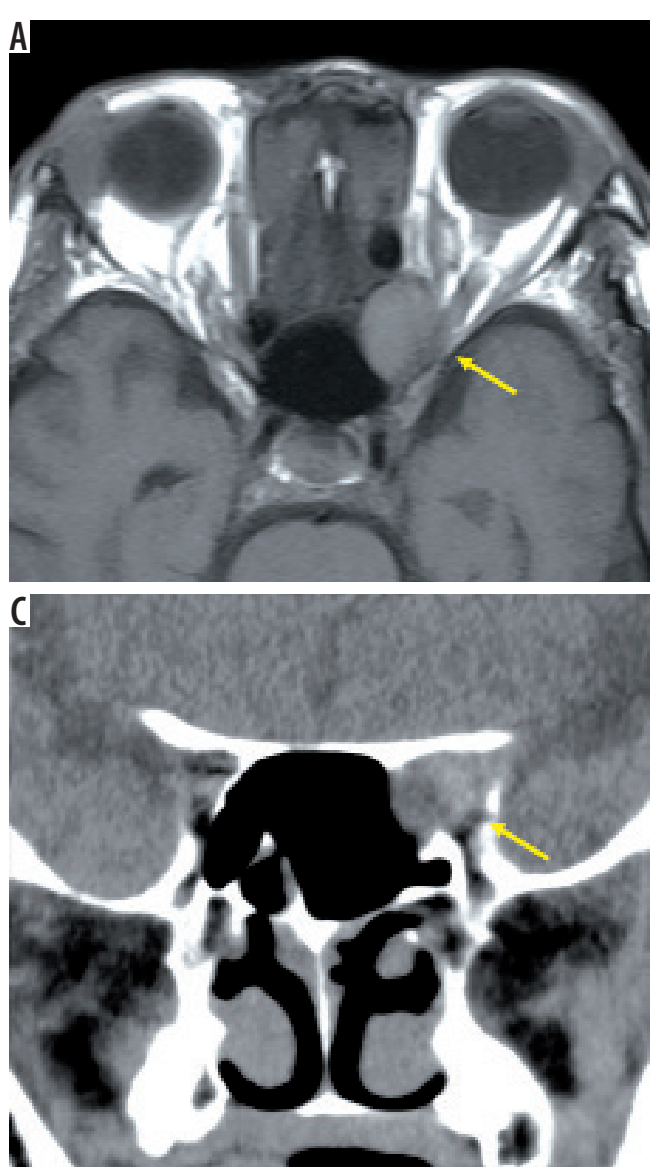

trast effect due to fluid containing a high level of protein (Figure 19) [49].

Thyroid ophthalmopathy is an orbital condition that has a strong association with thyroid autoimmune diseases, particularly Graves' disease. Dysthyroid optic neuropathy is the most severe form of thyroid ophthalmopathy, and the optic nerve is compressed by the enlarged extraocular muscles, resulting in visual impairment. Dysthyroid optic neuropathy is relatively rare and accounts for less than $5 \%$ of thyroid ophthalmopathy cases in most studies. MRI can show optic nerve compression by swollen extraocular muscles, and it can demonstrate inflam-

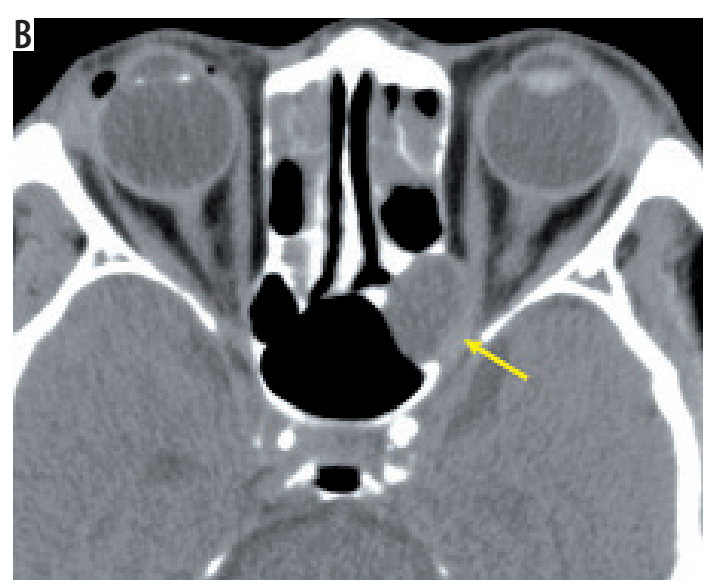

Figure 19. Mucocele in the Onodi cell. On the T1-weighted image (A) a high-signal mass with a clear border (yellow arrow) is located inside the optic canal. The surrounding bone is expanded and thinned on the axial CT (B). The optic canal is narrowed by the tumour (yellow arrow) on coronal CT (C)

mation of extraocular muscles and orbital fat tissue with high-intensity signal on STIR images. The optic nerve in dysthyroid optic neuropathy is almost normal on conventional MRI. Attempts have been made to detect narrowing of the optic nerve diameter, using high-resolution T1-weighted images, but the results overlap with thyroid ophthalmopathy without visual impairment [50].

\section{Conflict of interest}

The authors declare no conflict of interest.

\section{References}

1. Jäger HR, Miszkiel KA. Pathology of the optic nerve. Neuroimaging Clin N Am 2008; 18: 243-259.

2. Becker M, Masterson K, Delavelle J, Viallon M, Vargas MI, Becker CD. Imaging of the optic nerve. Eur J Radiol 2010; 74: 299-313.

3. Toosy AT, Mason DF, Miller DH. Optic neuritis. Lancet Neurol 2014; 13: 83-99

4. Optic Neuritis Study Group. Multiple sclerosis risk after optic neuritis: final optic neuritis treatment trial follow-up. Arch Neurol 2008; 65: 727-732.

5. Fatima Z, Motosugi U, Muhi A, Hori M, Ishigame K, Araki T. Diffusion-weighted imaging in optic neuritis. Can Assoc Radiol J 2013; 64: 51-55.
6. Thompson AJ, Banwell BL, Barkhof F, et al. Diagnosis of multiple sclerosis: 2017 revisions of the McDonald criteria. Lancet Neurol 2018; 17: 162-173.

7. Jarius S, Paul F, Aktas O, et al. MOG encephalomyelitis: international recommendations on diagnosis and antibody testing. J Neuroinflammation 2018; 15: 134.

8. Wingerchuk DM, Banwell B, Bennett JL, et al. International consensus diagnostic criteria for neuromyelitis optica spectrum disorders. Neurology 2015; 85: 177-189.

9. Krupp LB, Tardieu M, Amato MP, et al. International Pediatric Multiple Sclerosis Study Group criteria for pediatric multiple sclerosis and immune-mediated central nervous system demyelinating 
disorders: revisions to the 2007 definitions. Mult Scler 2013; 19: 1261-1267.

10. Daoud E, Chabchoub I, Neji H, et al. How MRI can contribute to the diagnosis of acute demyelinating encephalomyelitis in children. Neurosciences 2011; 16: 137-145.

11. Kale N. Optic neuritis as an early sign of multiple sclerosis. Eye Brain 2016; 8: 195-202.

12. Swanton JK, Fernando K, Dalton CM, et al. Is the frequency of abnormalities on magnetic resonance imaging in isolated optic neuritis related to the prevalence of multiple sclerosis? A global comparison. J Neurol Neurosurg Psychiatry 2006; 77: 1070-1072.

13. Mealy MA, Whetstone A, Orman G, Izbudak I, Calabresi PA, Levy M. Longitudinally extensive optic neuritis as an MRI biomarker distinguishes neuromyelitis optica from multiple sclerosis. J Neurol Sci 2015; 355 : 59-63.

14. Patterson SL, Goglin SE. Neuromyelitis Optica. Rheum Dis Clin North Am 2017; 43: 579-591.

15. Jarius S, Paul F, Aktas O, et al. MOG encephalomyelitis: international recommendations on diagnosis and antibody testing. J Neuroinflammation 2018; 15: 134.

16. Chen JJ, Flanagan EP, Jitprapaikulsan J, et al. Myelin oligodendrocyte glycoprotein antibody-positive optic neuritis: clinical characteristics, radiologic clues, and outcome. Am J Ophthalmol 2018; 195: 8-15.

17. Remond P, Attyé A, Lecler A, et al. The central bright spot sign: a potential new MR imaging sign for the early diagnosis of anterior ischemic optic neuropathy due to giant cell arteritis. AJNR Am J Neuroradiol 2017; 38: 1411-1415.

18. Duftner C, Dejaco C, Sepriano A, Falzon L, Schmidt WA, Ramiro S. Imaging in diagnosis, outcome prediction and monitoring of large vessel vasculitis: a systematic literature review and meta-analysis informing the EULAR recommendations. RMD Open 2018; 4: e000612.

19. Orssaud C, Roche O, Dufier JL. Nutritional optic neuropathies. J Neurol Sci 2007; 262: 158-164.

20. Lawton AW, Frisard NE. Visual loss, retinal hemorrhages, and optic disc edema resulting from thiamine deficiency following bariatric surgery complicated by prolonged vomiting. Ochsner J 2017; 17: 112-114.

21. Kerrison JB. Optic neuropathies caused by toxins and adverse drug reactions. Ophthalmol Clin North Am 2004; 17: 481-488.

22. Grzybowski A, Zülsdorff M, Wilhelm H, Tonagel F. Toxic optic neuropathies: an updated review. Acta Ophthalmol 2015; 93: 402-410.

23. Archer EL, Liao EA, Trobe JD. Radiation-induced optic neuropathy: clinical and imaging profile of twelve patients. J Neuroophthalmol 2019; 39: 170-180.

24. McKeever RG, Greenberg MI, Lange JR. Ischemic optic neuropathies. N Engl J Med 2015; 373: 1676-1677

25. He M, Cestari D, Cunnane MB, Rizzo JF. The use of diffusion MRI in ischemic optic neuropathy and optic neuritis. Semin Ophthalmol 2010; 25: 225-232.

26. Newman NJ, Biousse V. Hereditary optic neuropathies. Eye (Lond) 2004; 18: 1144-1160.

27. Blanc C, Heran F, Habas C, Bejot Y, Sahel J, Vignal-Clermont C. MRI of the optic nerves and chiasm in patients with leber hereditary optic neuropathy. J Neuroophthalmol 2018; 38: 434-437.

28. Papaliodis GN. Ophthalmologic manifestations of systemic vasculitis. Curr Opin Ophthalmol 2017; 28: 613-616.

29. Yang Q, Sun L, Wang Q, et al. Primary optic neuropathy in Behçet's syndrome. Mult Scler 2018 doi: 10.1177/1352458518786058.
30. Dammacco R. Systemic lupus erythematosus and ocular involvement: an overview. Clin Exp Med 2018; 18: 135-149.

31. Muhle C, Reinhold-Keller E, Richter C, et al. MRI of the nasal cavity, the paranasal sinuses and orbits in Wegener's granulomatosis. Eur Radiol 1997; 7: 566-570.

32. Takazawa T, Ikeda K, Nagaoka T, et al. Wegener granulomatosis-associated optic perineuritis. Orbit 2014; 33: 13-16.

33. André R, Cottin V, Saraux JL, et al. Central nervous system involvement in eosinophilic granulomatosis with polyangiitis (ChurgStrauss): Report of 26 patients and review of the literature. Autoimmun Rev 2017; 16: 963-969.

34. Chwalisz BK, Stone JH. Neuro-ophthalmic complications of IgG4-related disease. Curr Opin Ophthalmol 2018; 29: 485-494.

35. Groen F, Rothova A. Ocular involvement in sarcoidosis. Semin Respir Crit Care Med 2017; 38: 514-522

36. De Abreu MR, Chung CB, Biswal S, Haghighi P, Hesselink J, Resnick D. Erdheim-Chester disease: MR imaging, anatomic, and histopathologic correlation of orbital involvement. AJNR Am J Neuroradiol 2004; 25: 627-630.

37. Kansara S, Bell D, Johnson J, Zafereo M. Head and neck inflammatory pseudotumor: Case series and review of the literature. Neuroradiol J 2016; 29: 440-446.

38. Velayudhan V, Chaudhry ZA, Smoker WRK, Shinder R, Reede DL. Imaging of intracranial and orbital complications of sinusitis and atypical sinus infection: what the radiologist needs to know. Curr Probl Diagn Radiol 2017; 46: 441-451.

39. Chang YM, Chang YH, Chien KH, et al. Orbital apex syndrome secondary to aspergilloma masquerading as a paranasal sinus tumor: A case report and literature review. Medicine (Baltimore) 2018; 97: e11650.

40. Garg RK, Malhotra HS, Kumar N, Uniyal R. Vision loss in tuberculous meningitis. J Neurol Sci 2017; 375: 27-34.

41. Kumaran AM, Sundar G, Chye LT. Traumatic optic neuropathy: a review. Craniomaxillofac Trauma Reconstr 2015; 8: 31-41.

42. Henderson AD, Miller NR. Carotid-cavernous fistula: current concepts in aetiology, investigation, and management. Eye (Lond) 2018; 32: 164-172.

43. van der Poel NA, de Witt KD, van den Berg R, de Win MM, Mourits MP. Impact of superior ophthalmic vein thrombosis: a case series and literature review. Orbit 2019; 38: 226-232.

44. Tailor TD, Gupta D, Dalley RW, Keene CD, Anzai Y. Orbital neoplasms in adults: clinical, radiologic, and pathologic review. Radiographics 2013; 33: 1739-1758.

45. Singhal S, Birch JM, Kerr B, Lashford L, Evans DG. Neurofibromatosis type 1 and sporadic optic gliomas. Arch Dis Child 2002; 87: 65-70.

46. Olsen TG, Heegaard S. Orbital lymphoma. Surv Ophthalmol 2019; 64: 45-66.

47. Duthoy W, Boterberg T, Claus F, et al. Postoperative intensity-modulated radiotherapy in sinonasal carcinoma: clinical results in 39 patients. Cancer 2005; 104: 71-82.

48. Ahmad SM, Esmaeli B. Metastatic tumors of the orbit and ocular adnexa. Curr Opin Ophthalmol 2007; 18: 405-413.

49. Nickerson JP, Lane AP, Subramanian PS, Izbudak I. Onodi cell mucocele causing acute vision loss: radiological and surgical correlation. Clin Neuroradiol 2011; 21: 245-248.

50. Dodds NI, Atcha AW, Birchall D, Jackson A. Use of high-resolution MRI of the optic nerve in Graves' ophthalmopathy. Br J Radiol 2009; 82: 541-544. 